\title{
Valores de Referência da Tomografia de Coerência Óptica na Idade Pediátrica
}

\author{
Normative Database of Optical Coherence Tomography Parameters in \\ Childhood
}

\author{
Tatiana QUEIRÓS ${ }^{1}$, Cristina FREITAS ${ }^{1}$, Sandra GUIMARÃES ${ }^{1}$ \\ Acta Med Port 2015 Mar-Apr;28(2):148-157
}

\begin{abstract}
RESUMO
Introdução: A tomografia de coerência óptica é um exame que permite obter imagens de alta resolução dos tecidos in vivo, possibilitando a medição das estruturas oculares, nomeadamente a camada de fibras nervosas da retina e a espessura macular. Como método não invasivo torna-se particularmente útil em crianças, contudo a sua aplicabilidade está limitada pela existência de valores normativos apenas para adultos.
\end{abstract}

Objetivo: Estabelecer na idade pediátrica valores normativos para a espessura da camada de fibras nervosas da retina e espessura macular, averiguando a sua influência com o género, idade, refração, lateralidade e dominância ocular.

Material e Métodos: Foram submetidas a exame oftalmológico e a Cirrus HD-tomografia de coerência óptica (Carl Zeiss Meditec) 153 crianças dos quatro aos 17 anos.

Resultados: Obtiveram-se valores da espessura média global da camada de fibras nervosas da retina de 97,90 $\mu \mathrm{m}$. Não se detectaram diferenças entre géneros e com a idade, mas sim consoante a lateralidade e dominância ocular. Verificou-se um aumento da espessura com refrações positivas. Com o protocolo Macular Cube 512 × 128 verificou-se que o campo central apresentou a menor espessura $(250,35 \mu \mathrm{m})$, apresentando os rapazes maior espessura macular.

Discussão: Os valores da espessura da camada de fibras nervosas da retina e da espessura macular obtidos são comparáveis a estudos recentes. A distribuição da espessura por quadrantes respeita a distribuição normal da camada de fibras nervosas da retina. A espessura macular revelou-se superior no género masculino (campo central e anel interno), dados estes também concordantes com estudos prévios.

Conclusão: Estabelecemos as normativas da espessura da camada de fibras nervosas da retina e espessura macular em crianças portuguesas saudáveis, dados estes que reestruturam a avaliação e interpretação dos parâmetros obtidos pela tomografia de coerência óptica no diagnóstico de patologias pediátricas na prática clínica.

Palavras-chave: Criança; Fibras Nervosas; Macula Lutea; Retina; Tomografia de Coerência Óptica.

\section{ABSTRACT}

Introduction: Optical coherence tomography is a technology that allows obtaining high resolution images of tissues in vivo, enabling the measurement of ocular structures, including the retinal nerve fiber layer and macular thickness. As a noninvasive test it's particularly useful in children, but its applicability is limited by the existence of normative values for adults only.

Purpose: To establish the pediatric normative values of retinal nerve fiber layer thickness and macular thickness and to investigate its relationship with sex, age, refraction, eye side and ocular dominance.

Material and Methods: Ophthalmologic examination and Cirrus HD-optical coherence tomography (Carl Zeiss Meditec) were carried out on 153 children aged 4 to 17 years old.

Results: We obtained a mean retinal nerve fiber layer average thickness of $97.90 \mu \mathrm{m}$. No significant differences were detected between genders, however the eye side and ocular dominance had significant influence on retinal nerve fiber layer thickness. Retinal nerve fiber layer thickness increased significantly with more positive refraction. With the Macular Cube $512 \times 128$ protocol we found that the average central subfield showed the smallest thickness $(250.35 \mu \mathrm{m})$ and boys had higher macular thickness.

Discussion: The values of the retinal nerve fiber layer thickness and macular thickness obtained are comparable to recent studies. The distribution of retinal nerve fiber layer thickness in quadrants is in agreement with the normal distribution of retinal nerve fiber layer. Macular thickness proved to be higher in males (center field and inner ring), data consistent with previous studies.

Conclusion: We establish the normative retinal nerve fiber layer thickness and macular thickness in healthy Portuguese children. These data enhance the evaluation and interpretation of parameters obtained by optical coherence tomography in the diagnosis of pediatric disorders in clinical practice.

Keywords: Child; Macula Lutea; Nerve Fibers; Retina; Tomography, Optical Coherence.

\section{INTRODUÇÃO}

A tomografia de coerência óptica (OCT), primeiramente descrita por Huang et al em 1991, é um exame de imagem que usa interferometria de baixa coerência para determinar o tempo de eco e a magnitude da reflectividade da luz num objeto em estudo. ${ }^{1}$ Obtêm-se assim imagens tridimensionais, em plano transversal, de muito alta resolução (3 a 15 $\mu \mathrm{m})$ dos tecidos in vivo, permitindo a medição das estrutu- ras oculares, incluindo córnea, retina, camada de fibras nervosas da retina (CFNR), mácula e disco óptico. ${ }^{2}$ Por todos estes motivos, a OCT emergiu para a vanguarda da tecnologia da imagem ocular, sendo atualmente um importante procedimento de diagnóstico e de seguimento de neuropatias ópticas, patologias retinianas, anomalias do nervo óptico, edema macular, buraco macular, entre outras. ${ }^{2-5}$

1. Serviço de Oftalmologia. Hospital de Braga. Braga. Portugal.

Recebido: 27 de Janeiro de 2015 - Aceite: 09 de Março de 2015 | Copyright @ Ordem dos Médicos 2015 
A nova geração de OCT, o spectral-domain OCT (SD-OCT), permite maior resolução, melhor qualidade e menor tempo de aquisição de imagem (40 a 110 vezes mais rápido que o modelo anterior - time-domain OCT), com menor possibilidade de ocorrência de artefactos associados ao movimento ocular. ${ }^{2,4,6}$

Como método não invasivo, rápido, inócuo e sem contacto direto ocular, a OCT é particularmente útil em crianças, $^{7,8}$ estando já estabelecido a sua exequibilidade e reprodutibilidade nesta população. ${ }^{8-10}$ Contudo, a sua aplicabilidade na idade pediátrica é limitada por só existirem valores normativos para indivíduos com 18 anos ou mais.

Os dados literários sobre normativas em crianças são escassos $^{3,5,7,8,10-14}$ e são ainda menos os que utilizam o SD-OCT.,14-17 Tanto quanto foi possível apurar, não existem artigos publicados que estudem, sectorialmente e em simultâneo, os valores normativos da espessura da CFNR e espessura macular determinados pelo SD-OCT numa população caucasiana de crianças entre os quatro e os 17 anos de idade.

Assim, o objetivo deste estudo é estabelecer as normativas para a espessura da CFNR e espessura macular em crianças portuguesas saudáveis, averiguando a sua influência com o género, idade, refração, lateralidade e dominância ocular.

\section{MATERIAL E MÉTODOS \\ População de estudo}

Este foi um estudo observacional, transversal e analítico realizado no Serviço de Oftalmologia do Hospital de Braga, durante seis meses consecutivos, após a autorização do Diretor de Serviço de Oftalmologia e parecer favorável da Comissão Executiva e da Comissão de Ética do respetivo hospital. A anuência em participar no estudo foi feita através de consentimento verbal da criança e assinatura do consentimento informado pelo responsável legal.

A população em estudo abrange crianças caucasianas com idades entre os quatro e os 17 anos, inclusive, que frequentaram a consulta de Oftalmologia e que cumprissem os critérios de inclusão, nomeadamente, acuidade visual corrigida monocular de, pelo menos, 10/10 e erro refrativo, em equivalente esférico (ES), entre -5 e +5 dioptrias. Os critérios de exclusão foram os seguintes: prematuridade; história ou evidência de ambliopia ou estrabismo; história familiar de glaucoma; alterações do disco óptico sugestivas de glaucoma (rácio escavação-disco $\geq 0,5 \mathrm{em}$ qualquer dos olhos; assimetria entre os rácios dos dois olhos $\geq 0,2$; hemorragia) ou qualquer outra alteração observada na oftalmoscopia direta ou na lâmpada de fenda; cirurgia ocular prévia; trauma ocular prévio; e atraso no desenvolvimento psicomotor.

Todas as crianças elegíveis foram submetidas a exame oftalmológico, por especialista em oftalmologia, incluindo: avaliação ortóptica; biomicroscopia com lâmpada de fenda; e fundoscopia sem dilatação pupilar. A acuidade visual corrigida de cada criança foi determinada pela tabela de Snellen, com ou sem correção óptica conforme necessidade.
O erro refrativo foi avaliado por refração subjetiva efetuada após medição com autorrefractómetro (Topcon KR-8900). No caso de crianças não cooperantes ou quando clinicamente necessário, foi induzida cicloplegia por administração de cloridrato de ciclopentolato $10 \mathrm{mg} / \mathrm{ml}$ num total de três gotas, com intervalos de cinco minutos. Após um mínimo de 40 minutos da última gota foi medido o erro refrativo. Para análise, o erro refrativo foi registado como ES, calculado pela seguinte fórmula: $E S=$ esfera $+1 / 2$ cilindro. $A$ identificação do olho dominante baseou-se no teste de Dolman (hole-in-card test). ${ }^{18} \mathrm{~A}$ informação relativa ao género, idade, antecedentes familiares e pessoais foi recolhida pela consulta dos processos clínicos electrónicos.

\section{SD-OCT}

A espessura da CFNR e espessura macular foram obtidas por exame de SD-OCT (Cirrus HD-OCT, model 4000; Carl Zeiss Meditec). Os exames foram realizados sem dilatação pupilar, pois as características ópticas e de velocidade de aquisição deste modelo permitem obter imagens de qualidade com um diâmetro pupilar a partir de $2,5 \mathrm{~mm} .{ }^{19}$ Todos os exames foram realizados pela mesma pessoa (TQ) e com o mesmo instrumento, efetuando-se três medições de cada protocolo em cada olho e registando-se para análise o valor médio.

O protocolo Optic Disc Cube 200x200 foi utilizado para determinar a espessura da CFNR (espessura média global, espessura por quadrante - superior, temporal, inferior e nasal - e espessuras individuais de 12 sectores de $30^{\circ}$ ) e o protocolo Macular Cube 512x128 para determinar a espessura e o volume macular. Este protocolo exibe também a espessura média de nove sectores formados por três círculos de diâmetros de $1 \mathrm{~mm}, 3 \mathrm{~mm}$ e $6 \mathrm{~mm}$, divididos, à exceção do círculo central, em quatro quadrantes: superior, nasal, inferior e temporal. ${ }^{19}$

Nos dois protocolos e em todos os casos, utilizou-se o método interno de fixação do alvo e a centralização do scan foi assegurada por observação direta do fundo ocular no ecrã. Realça-se que todos os scans com força de sinal $<5$, mal centrados, com artefactos de movimentos ou incompletos foram excluídos.

\section{Análise estatística}

Toda a análise estatística foi realizada por recurso ao programa Statistical Package for the Social Sciences versão 20 (IBM SPSS Statistics v.20.0). Após verificada a condição da normalidade dos dados pelo teste de Kolmogorov-Smirnov e pelas medidas de assimetria e curtose, efetuou-se: estatística descritiva para caracterização da amostra; determinação das medidas de tendência central e de dispersão e definição dos percentis 99, 95, 5 e 1 para todos os parâmetros obtidos na OCT; comparação das variáveis entre olho direito e esquerdo, assim como entre olho dominante e olho não dominante, através do $t$-test para amostras emparelhadas; comparação das variáveis entre géneros através do $t$-test para amostras independentes; comparação entre os quadrantes da CFNR e as regiões 
maculares através do teste ANOVA de medições repetidas com correção de Bonferroni para identificar quais os pares de médias que diferem significativamente entre si; e, por fim, regressão linear múltipla para obter um modelo que permita predizer o valor da espessura da CFNR e da espessura macular (variáveis dependentes) em função do género, idade e erro refractivo (variáveis independentes).

Os resultados serão apresentados como média \pm desvio padrão (DP) e considerou-se significância estatística para valores de $p \leq 0,05$ (intervalo de confiança de 95\%).

\section{RESULTADOS}

\section{Caracterização da amostra}

Realizaram a OCT 164 crianças, das quais 11 foram posteriormente excluídas da análise por não ter sido possível obter imagens de qualidade. Por conseguinte, obteve-se uma amostra final de 306 olhos de 153 crianças. Destas, 82 crianças eram do género feminino $(53,6 \%)$ e 71 do género masculino (46,4\%), com média de idades de 9,54 $\pm 3,35$, sem diferença estatisticamente significativa entre o género e a média de idades $(p=0,310)$. A dominância ocular, identificada em todas as crianças, foi preponderantemente atribuída ao olho direito $(59,5 \%)$. A média do ES desta amostra foi de $-0,39 \pm 1,33$ dioptrias, sem diferenças estatisticamente significativas entre géneros $(p=0,242)$, lateralidade e dominância ocular $(p=0,937)$.

O estudo da espessura da CFNR foi efetuado em 140 crianças $(91,5 \%)$ e o estudo da espessura macular em 152 crianças $(99,3 \%)$, tendo em conta que nalgumas das crianças mais jovens foi possível obter o protocolo macular, mas não o do disco óptico e numa criança aconteceu o contrário.

\section{Análise da espessura da CFNR}

A espessura média global, por quadrantes e pelos 12 sectores da CFNR obtidos pela OCT estão representados na tabela 1. A espessura média global da CFNR na totalidade dos olhos foi de $97,90 \pm 9,32 \mu \mathrm{m}$, observando-se diferenças de magnitude elevada e estatisticamente significativas entre os quadrantes $(p<0,001)$. De facto, o valor médio da espessura da CFNR no quadrante inferior $(129,58 \pm 15,05$ $\mu \mathrm{m})$ e superior $(126,91 \pm 16,51 \mu \mathrm{m})$ foram superiores à espessura da CFNR no quadrante nasal $(69,72 \pm 10,47 \mu \mathrm{m})$ e temporal $(65,17 \pm 8,91 \mu \mathrm{m}$ ) (todos os $p<0,05$ ). Não se verificaram diferenças estatisticamente significativas entre os dois primeiros quadrantes $(p=0,238)$, mas sim entre $o$ quadrante nasal e temporal $(p<0,001)$, sendo este último o que apresenta menor espessura.

No que diz respeito à distribuição da espessura da CFNR por género é de salientar que não se encontraram diferenças estatisticamente significativas (Tabela 2). A distribuição da espessura da CFNR consoante a lateralidade e dominância ocular (Tabela 2) demostra que o olho esquerdo apresenta maior espessura no quadrante superior comparativamente ao olho direito $(p=0,0055)$ e, em contrapartida, no quadrante temporal observa-se uma espessura superior no olho direito $(p<0,001)$. No quadrante temporal verifica-se ainda que o olho dominante apresenta maior espessura da CFNR em relação ao olho não dominante $(p=$ 0,0145).

A análise da regressão linear múltipla (Tabela 3) permite identificar o ES como preditor significativo da espessura média global da CFNR $(\beta=0,218 ; p=0,011)$ e da espessura da CFNR no quadrante superior $(\beta=0,217 ; p=0,012)$ e inferior $(\beta=0,203 ; p=0,018)$. Esta variável apresenta uma relação positiva com estes parâmetros da CFNR, verificando-se um aumento da espessura da CFNR com

Tabela 1 - Distribuição dos parâmetros da espessura da CFNR avaliados nas 140 crianças (280 olhos)

\begin{tabular}{|c|c|c|c|c|c|}
\hline $\begin{array}{c}\text { Espessura CFNR } \\
(\mu \mathrm{m})\end{array}$ & Média (DP) & P99 & P95 & P5 & P1 \\
\hline Média global & $97,90(9,32)$ & 124,34 & 112,00 & 83,08 & 73,55 \\
\hline QS & $126,91(16,51)$ & 186,72 & 156,42 & 104,53 & 85,26 \\
\hline Q & $129,58(15,05)$ & 166,75 & 156,40 & 104,53 & 95,28 \\
\hline QT & $65,17(8,91)$ & 88,95 & 79,48 & 50,50 & 47,71 \\
\hline QN & $69,72(10,47)$ & 98,59 & 91,30 & 54,03 & 48,41 \\
\hline Sector 1 & $118,62(20,04)$ & 169,63 & 150,00 & 90,00 & 67,88 \\
\hline Sector 2 & $85,56(16,89)$ & 135,27 & 118,92 & 60,58 & 54,21 \\
\hline Sector 3 & $52,97(8,65)$ & 76,30 & 69,87 & 40,05 & 35,23 \\
\hline Sector 4 & $68,08(12,82)$ & 97,77 & 93,92 & 46,55 & 42,91 \\
\hline Sector 5 & $108,86(20,96)$ & 170,17 & 145,50 & 76,03 & 67,91 \\
\hline Sector 6 & $143,70(22,73)$ & 202,30 & 181,90 & 109,53 & 86,71 \\
\hline Sector 7 & $136,34(21,55)$ & 184,84 & 171,35 & 101,03 & 87,35 \\
\hline Sector 8 & $68,61(12,85)$ & 107,65 & 92,98 & 50,10 & 42,73 \\
\hline Sector 9 & $50,25(6,17)$ & 66,89 & 60,50 & 40,03 & 37,62 \\
\hline Sector 10 & $76,59(12,51)$ & 118,86 & 95,00 & 59,03 & 53,00 \\
\hline Sector 11 & $130,48(22,44)$ & 203,51 & 168,37 & 93,00 & 79,41 \\
\hline Sector 12 & $131,68(26,76)$ & 212,89 & 181,17 & 91,03 & 65,56 \\
\hline
\end{tabular}




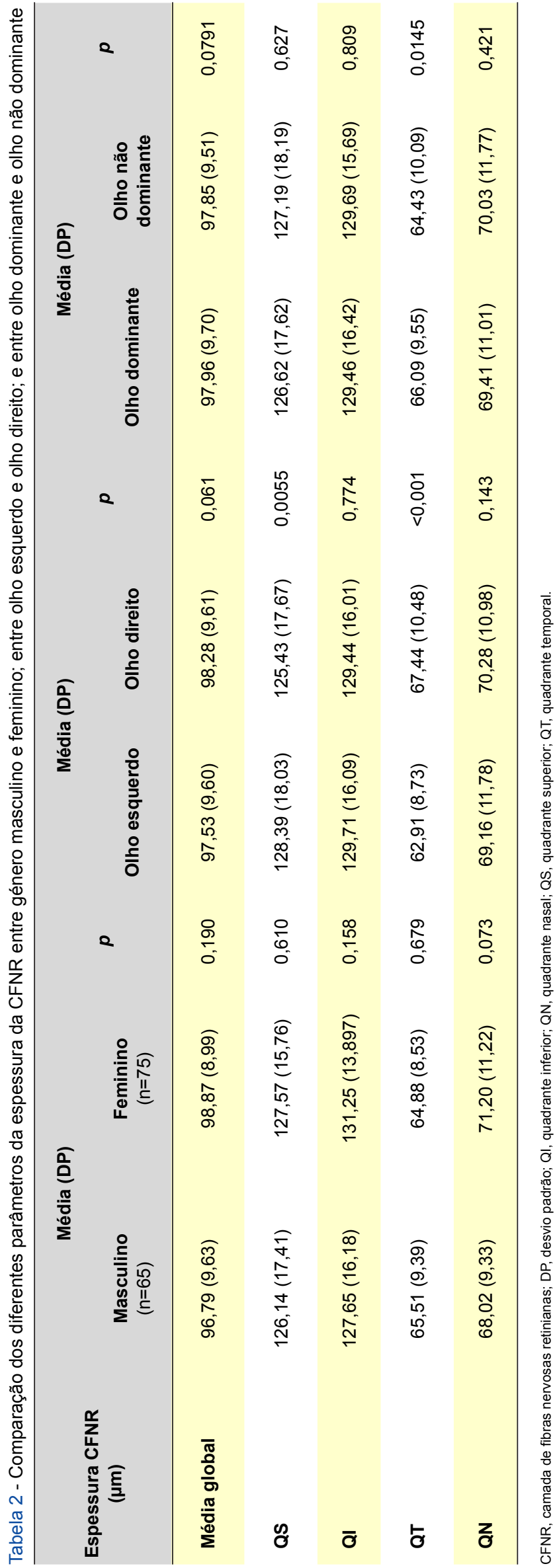

refrações positivas. Constata-se que a espessura média global, a espessura no quadrante superior e no quadrante inferior aumentam em média 1,6 $\mu \mathrm{m}, 2,8 \mu \mathrm{m}$ e 2,4 $\mu \mathrm{m}$, respetivamente, por cada dioptria positiva no ES. O género e a idade não apresentaram efeito significativo na predição da espessura de qualquer um dos parâmetros da CFNR obtidos pela OCT.

\section{Análise da espessura macular}

A média global da espessura macular, do campo central e dos quatro quadrantes do anel interno e externo, assim como do volume total obtidos pela OCT estão representados na Tabela 4. Constatam-se diferenças de magnitude elevada e estatisticamente significativas entre as diferentes áreas maculares $(p<0,001)$, sendo o campo central o que apresenta menor espessura $(250,35 \pm 19,28 \mu \mathrm{m})$, seguido da espessura média do anel externo $(279,91 \pm 12,16$ $\mu \mathrm{m})$. Por sua vez, o anel interno é a região que apresenta maior espessura macular $(316,03 \pm 14,05 \mu \mathrm{m})$, verificando-se que esta tendência se mantém quando se compara os quadrantes correspondentes (todos os $p<0,05$ ). Por outro lado, quando se compara entre si os quadrantes do mesmo anel, verifica-se a seguinte ordem crescente de espessura: quadrante temporal <inferior < superior <nasal (todos os $p<0,05)$. Estas diferenças são de magnitude elevada e estatisticamente significativas quer na região interna $(p<$ $0,001)$, quer na região externa macular $(p<0,001)$.

A estratificação por género (Tabela 5 ) revela que, de modo estatisticamente significativo, o género masculino apresenta maior espessura macular ao nível do campo central $(p=0,0135)$ e ao nível do anel interno, quer na sua média global $(p=0,01)$, quer nos quadrantes inferior $(p=$ $0,015)$, temporal $(p=0,001)$ e nasal $(p=0,0125)$. À semeIhança da CFNR, verifica-se que o olho esquerdo apresenta maior espessura no quadrante superior do anel externo macular comparativamente ao olho direito $(p=0,024)$. De igual modo, o quadrante temporal apresenta uma espessura superior no olho direito, quer no anel interno ( $p=$ $0,0025)$, quer no anel externo macular $(p=0,0115)$ (Tabela 5 ). No que diz respeito à distribuição da espessura macular consoante a dominância ocular, não se encontraram diferenças estatisticamente significativas em nenhum dos parâmetros avaliados (Tabela 5).

Pela análise da regressão linear múltipla (Tabela 6) constata-se que a espessura macular no campo central apresenta como preditores significativos o género $(\beta=$ $-0,214 ; p=0,006)$, a idade $(\beta=0,187 ; p=0,019)$ e o ES $(\beta=-0,204 ; p=0,011)$. Verifica-se uma diminuição em média da espessura de $8 \mu \mathrm{m}$ no género feminino, sendo esta a variável independente que apresenta maior contribuição relativa. O ES apresenta também uma relação negativa com a espessura do campo central, verificando-se uma diminuição da espessura com refrações positivas (diminuição em média de $3 \mu \mathrm{m}$ por cada dioptria positiva no ES). A idade, por outro lado, apresenta uma relação positiva com este parâmetro, constatando-se um aumento da espessura em média de $1 \mu \mathrm{m}$ por ano. $O$ género é também preditor 
Tabela 3 - Resultados da regressão linear múltipla com os diferentes parâmetros da espessura da CFNR como variáveis dependentes

\begin{tabular}{|c|c|c|c|c|c|c|c|c|}
\hline $\begin{array}{c}\text { Espessura CFNR } \\
\qquad(\mu \mathrm{m})\end{array}$ & VI & $F(3,136)$ & B & beta $(\beta)$ & LS & LI & $p$ & $\mathbf{R}^{2}{ }_{\text {AJ }}$ \\
\hline \multirow{3}{*}{ Média global } & Género & \multirow{3}{*}{$\begin{array}{c}4,176 \\
p=0,007\end{array}$} & 2,262 & 0,122 & 5,284 & $-0,761$ & 0,141 & \multirow{3}{*}{0,064} \\
\hline & Idade & & $-0,331$ & $-0,115$ & 0,147 & $-0,808$ & 0,174 & \\
\hline & ES & & 1,587 & 0,218 & 2,801 & 0,373 & 0,011 & \\
\hline \multirow{3}{*}{ QS } & Género & \multirow{3}{*}{$\begin{array}{c}3,136 \\
p=0,015\end{array}$} & 1,766 & 0,054 & 7,154 & $-3,621$ & 0,518 & \multirow{3}{*}{0,053} \\
\hline & Idade & & $-0,587$ & $-0,116$ & 0,264 & $-1,439$ & 0,175 & \\
\hline & ES & & 2,800 & 0,217 & 4,964 & 0,636 & 0,012 & \\
\hline \multirow{3}{*}{ Q } & Género & \multirow{3}{*}{$\begin{array}{c}3,709 \\
p=0,013\end{array}$} & 3,884 & 0,129 & 8,792 & $-1,024$ & 0,120 & \multirow{3}{*}{0,055} \\
\hline & Idade & & $-0,474$ & $-0,103$ & 0,300 & $-1,252$ & 0,227 & \\
\hline & ES & & 2,396 & 0,203 & 4,367 & 0,425 & 0,018 & \\
\hline \multirow{3}{*}{ QT } & Género & \multirow{3}{*}{$\begin{array}{c}0,660 \\
p=0,578\end{array}$} & ---- & ---- & ---- & ---- & ---- & \multirow{3}{*}{---} \\
\hline & Idade & & ---- & --- & ---- & ---- & ---- & \\
\hline & ES & & ---- & --- & --- & ---- & ---- & \\
\hline \multirow{3}{*}{ QN } & Género & \multirow{3}{*}{$\begin{array}{c}1,961 \\
p=0,123\end{array}$} & --- & ---- & ---- & --- & ---- & \multirow{3}{*}{---- } \\
\hline & Idade & & --- & ---- & --- & --- & ---- & \\
\hline & ES & & --- & ---- & --- & --- & ---- & \\
\hline
\end{tabular}

--- modelo ajustado não significativo $(p>0,05)$

CFNR, camada de fibras nervosas retinianas; ES, equivalente esférico; IC, intervalo de confiança; LS, limite superior; LI, limite inferior; QI, quadrante inferior; QN, quadrante nasal; QS, quadrante superior; QT, quadrante temporal; VI, variáveis independentes.

Tabela 4 - Distribuição dos parâmetros da espessura e volume maculares avaliados nas 152 crianças (304 olhos)

\begin{tabular}{|c|c|c|c|c|c|}
\hline $\begin{array}{l}\text { Espessura Macular } \\
\qquad(\mu \mathrm{m})\end{array}$ & Média (DP) & P99 & P95 & P5 & P1 \\
\hline EM global & $282,26(11,59)$ & 314,05 & 300,35 & 263,13 & 258,00 \\
\hline CC & $250,35(19,28)$ & 292,03 & 283,70 & 217,80 & 207,83 \\
\hline \multicolumn{6}{|l|}{ Anel Interno } \\
\hline Superior & $320,31(14,36)$ & 353,91 & 347,18 & 295,50 & 289,77 \\
\hline Inferior & $316,55(14,38)$ & 347,71 & 341,18 & 293,50 & 283,27 \\
\hline Temporal & $305,87(14,15)$ & 338,91 & 330,50 & 282,33 & 283,27 \\
\hline Nasal & $321,38(14,99)$ & 357,94 & 348,50 & 299,33 & 290,27 \\
\hline \multicolumn{6}{|l|}{ Anel Externo } \\
\hline Superior & $283,25(13,18)$ & 322,72 & 306,50 & 261,15 & 252,56 \\
\hline Inferior & $273,01(14,40)$ & 323,21 & 295,20 & 249,98 & 243,77 \\
\hline Temporal & $263,09(12,34)$ & 298,97 & 283,68 & 244,50 & 240,77 \\
\hline Nasal & $300,29(14,33)$ & 332,82 & 322,50 & 274,98 & 259,98 \\
\hline \multicolumn{6}{|l|}{ Volume $\left(\mathrm{mm}^{3}\right)$} \\
\hline Volume total & $10,17(0,41)$ & 11,31 & 10,82 & 9,47 & 9,28 \\
\hline
\end{tabular}

significativo da espessura global macular do anel interno $(\beta$ $=-0,204 ; p=0,012)$ e respetivos quadrantes temporal $(\beta=$ $-0,268 ; p=0,001)$ e nasal $(\beta=-0,204 ; p=0,012)$. Apresenta igualmente uma relação negativa com estes parâmetros da EM, verificando-se que no género feminino a espessura média global no anel interno e respetivos quadrantes temporal e nasal diminuem em média $5,7 \mu \mathrm{m}, 7,6 \mu \mathrm{m}$ e $6 \mu \mathrm{m}$, respetivamente. Verificou-se ainda que a idade é preditor significativo da espessura macular no quadrante superior do anel externo $(\beta=-0,177 ; p=0,033)$, apresentando uma relação negativa com este parâmetro. Nos restantes parâmetros da espessura macular não foi encontrada influência significativa com nenhuma das variáveis independentes (género, idade e ES).

\section{DISCUSSÃO}

$\mathrm{Na}$ idade pediátrica a OCT revela-se especialmente útil, permitindo o diagnóstico e seguimento de neuropatias ópticas, patologias retinianas, anomalias do nervo óptico e até mesmo lesões intracranianas. ${ }^{15,20,21}$ Afirma-se como método exequível nesta população, ,710,14 o que é suportado pelo presente estudo onde foi possível obter imagens de 


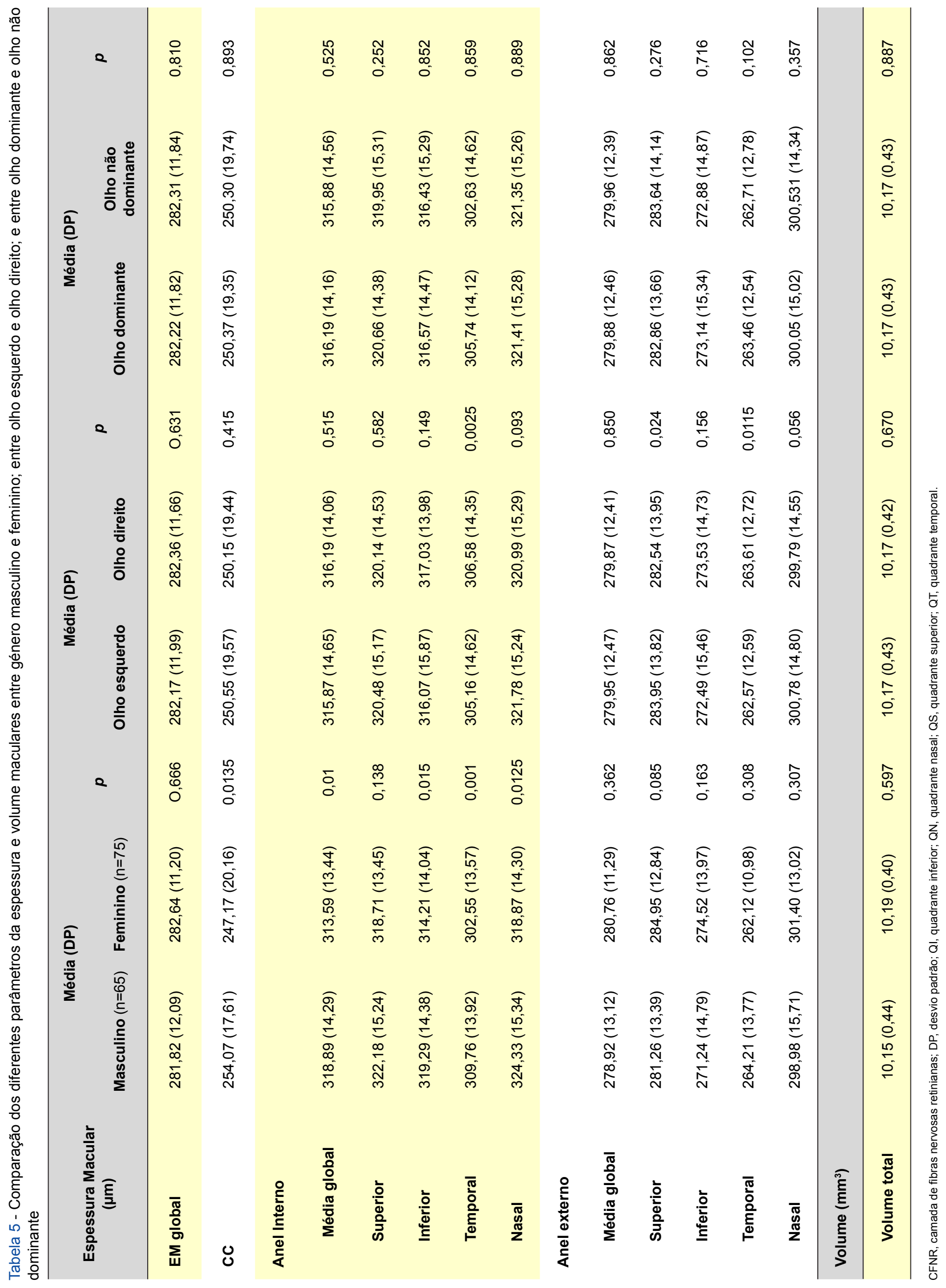


qualidade em $93,3 \%$ das crianças estudadas. Contudo a sua aplicabilidade na prática clínica actual é limitada pelo facto de só existirem normativas para a idade adulta.

A maioria da literatura disponível sobre valores normativos dos parâmetros da OCT em crianças recorre a outros modelos de OCT que, pelos diferentes algoritmos de aquisição de imagem e resolução axial, tornam as medições não intercambiáveis. ${ }^{22-24}$ Mais recentemente, Barrio-Barrio et al, e Al-Haddad et al, reportaram normativas da espessura da CFNR e espessura macular por recurso a Cirrus OCT em crianças caucasianas. O nosso estudo complementa estes ao reportar valores normativos de outros parâmetros da espessura da CFNR, nomeadamente a espessura pelos 12 sectores individuais; e averiguar a distribuição da espessura da CFNR e espessura macular consoante a lateralidade e dominância ocular.

Os valores da espessura da CFNR das 140 crianças envolvidas neste trabalho são comparáveis aos estudos recentes que utilizaram Cirrus OCT. ${ }^{9,15-17} \mathrm{~A}$ distribuição da espessura por quadrantes (inferior $>$ superior $>$ nasal $>$ temporal), anteriormente reportada em crianças, ${ }^{3,9,10,14-17}$ respeita a distribuição normal da CFNR em torno do nervo óptico, também conhecida por ISNT rule ou configuração em double hump. Esta configuração é o resultado do maior número de fibras que convergem para a cabeça do nervo óptico a partir das arcadas superior e inferior, em relação ao número de fibras que convergem a partir do feixe papilo-macular e retina nasal. ${ }^{3}$ Demostramos a assimetria da CFNR consoante a lateralidade, verificando-se, à semelhança de estudos prévios em crianças $^{8,25}$ e adultos ${ }^{1,26}$ que o olho esquerdo apresenta maior espessura no quadrante superior $\mathrm{e}$ que o olho direito apresenta maior espessura no quadrante temporal, sendo que o mecanismo fisiológico subjacente ainda não está estabelecido. A dominância ocular, atribuída preponderantemente ao olho direito, tal como descrito na literatura, ${ }^{18}$ teve influência nos resultados, verificando-se maior espessura da CFNR no quadrante temporal do olho dominante. Não foram encontradas outras referências que averiguassem esta relação por quadrantes, sendo assim necessário estudos posteriores para confirmar estes resultados. A influência do erro refractivo é discutível, havendo estudos que não encontram nenhuma relação ${ }^{27,28} \mathrm{e}$ outros, ${ }^{3,8,10,16,17}$ que à semelhança do atual, verificam uma diminuição da espessura da CFNR com refrações negativas. A explicação para este fenómeno não é consensual, propondo-se que o verdadeiro diâmetro do scan circular num olho míope (com maior comprimento axial) é maior que o pré-definido, resultando numa diminuição da medição da espessura da CFNR. ${ }^{29}$ Tal facto pode traduzir um artefacto de medição e não uma verdadeira alteração estrutural. Independentemente da explicação, é concebível que, sendo a OCT um método óptico, o erro refractivo possa afetar as suas medições, motivo pelo qual foram excluídas crianças com ES> \pm 5 dioptrias. Não se reportaram diferenças nos parâmetros da CFNR consoante o género, tal como a maioria dos estudos,,$^{10,14-17}$ não apresentando esta variável efeito significativo na predição da espessura. A idade tam- bém não apresentou efeito significativo, o que é concordante com a literatura. 4,7,10,12,14-17 Os resultados encontrados são comparáveis a estudos em adultos realizados com o Cirrus, ${ }^{22}$ o que é compreensível já que só se verifica uma diminuição significativa da espessura da CFNR após os 50 anos de idade. ${ }^{15}$ Esta diminuição não linear com a idade e a estreita faixa etária das crianças envolvidas nos diferentes estudos pode explicar a não associação encontrada entre estes parâmetros, sendo necessário estudos longitudinais para clarificar este tema.

O estudo da espessura macular foi realizado em número superior por ser um protocolo mais fácil de realizar, devido à tendência das crianças jovens olharem para o alvo localizado no centro da fóvea. Os valores da espessura macular das 152 crianças envolvidas são comparáveis aos estudos que utilizaram Cirrus OCT. 9,16,17,30 Topograficamente, e de modo concordante com outras referências, ${ }^{5,11,13,16,17}$ o anel interno apresentou a maior espessura macular e, na comparação por quadrantes, o temporal apresentou a menor espessura nas duas regiões. Tal como em estudos prévios, ,, 11,13,16,17,31 a distribuição da espessura macular por quadrantes ao nível do anel externo (temporal< inferior< superior< nasal) é consistente com a convergência das fibras nervosas retinianas no disco óptico. A espessura macular revelou-se superior no género masculino ao nível do campo central e anel interno, mas não ao nível do anel externo, dados estes concordantes com estudos prévios em crianças ${ }^{4,5,11,16,17}$ e adultos. ${ }^{31,32} \mathrm{~A}$ explicação para este fenómeno pode residir nas camadas retinianas externas à CFNR, as quais constituem a maior parte da espessura macular nestas regiões. ${ }^{31}$ Não obstante, a camada responsável por esta diferença ainda não foi identificada. $\mathrm{O}$ impacto do género na espessura macular foi ainda realçado na análise de regressão linear múltipla onde esta variável se revelou o preditor mais forte ao nível do campo central. A distribuição da espessura macular consoante a lateralidade revelou diferenças compatíveis com as encontradas para a CFNR, nomeadamente uma maior espessura macular no quadrante superior externo do olho esquerdo e no quadrante temporal interno e externo do olho direito. Apenas um estudo com o time-domain OCT em crianças de 6 anos averigua esta assimetria, detectando apenas resultado idêntico ao nível do quadrante superior. ${ }^{25} \mathrm{~A}$ diferença nas idades e versões de OCT utilizadas pode explicar os diferentes resultados encontrados. Não foram encontradas diferenças em nenhum dos parâmetros consoante a dominância ocular. Samarawickrama et al apenas estudou as diferenças ao nível do campo central mas de igual modo não reportou assimetria. ${ }^{33}$ Pelo recurso à regressão linear múltipla verificou-se um aumento da espessura macular no campo central com refrações negativas, tal como em estudos prévios em crianças ${ }^{5}$ e adultos. ${ }^{34,35} \mathrm{Wu}$ et al propõem que a tendência para o alongamento e achatamento da MLI resultaria numa elevação da fóvea e consequentemente maior espessura. ${ }^{35} \mathrm{O}$ facto desta área anatómica ser avascular, torna-a mais suscetível à deformação, o que suporta a hipótese supramencionada. ${ }^{5}$ Estes dados devem 
Tabela 6 - Resultados da regressão linear múltipla com os diferentes parâmetros da espessura e volume maculares como variáveis dependentes

\begin{tabular}{|c|c|c|c|c|c|c|c|c|}
\hline $\begin{array}{c}\text { Espessura CFNR } \\
(\mu \mathrm{m})\end{array}$ & VI & $F(3,136)$ & B & $\beta$ & LS & LI & $p$ & $\mathbf{R}_{\mathrm{AJ}}^{2}$ \\
\hline \multirow{3}{*}{ EM global } & Género & \multirow{3}{*}{$\begin{array}{c}0,421 ; \\
p=0,739\end{array}$} & ---- & ---- & ---- & ---- & ---- & \multirow{3}{*}{---- } \\
\hline & Idade & & ---- & --- & ---- & ---- & ---- & \\
\hline & ES & & ---- & ---- & ---- & ---- & ---- & \\
\hline \multirow{3}{*}{ CC } & Género & \multirow{3}{*}{$\begin{array}{c}7,057 \\
p<0,001\end{array}$} & $-8,241$ & $-0,214$ & $-2,346$ & $-14,137$ & 0,006 & \multirow{3}{*}{0,107} \\
\hline & Idade & & 1,074 & 0,187 & 1,972 & 0,176 & 0,019 & \\
\hline & ES & & $-2,955$ & $-0,204$ & $-0,687$ & $-5,224$ & 0,011 & \\
\hline \multicolumn{9}{|l|}{ Anel Interno } \\
\hline \multirow{3}{*}{ Média global } & Género & \multirow{3}{*}{$\begin{array}{c}2,947 \\
p=0,035\end{array}$} & $-5,735$ & $-0,204$ & $-1,274$ & $-10,195$ & 0,012 & \multirow{3}{*}{0,037} \\
\hline & Idade & & 0,496 & 0,119 & 1,176 & $-0,184$ & 0,151 & \\
\hline & ES & & $-0,638$ & $-0,060$ & 1,078 & $-2,355$ & 0,464 & \\
\hline \multirow{3}{*}{ Superior } & Género & \multirow{3}{*}{$\begin{array}{c}1,794 \\
p=0,151\end{array}$} & ---- & ---- & ---- & ---- & --- & \multirow{3}{*}{---- } \\
\hline & Idade & & --- & --- & --- & --- & --- & \\
\hline & ES & & --- & ---- & ---- & --- & ---- & \\
\hline \multirow{3}{*}{ Inferior } & Género & \multirow{3}{*}{$\begin{array}{c}2,291 \\
p=0,081\end{array}$} & ---- & ---- & ---- & ---- & ---- & \multirow{3}{*}{---- } \\
\hline & Idade & & --- & --- & --- & --- & --- & \\
\hline & ES & & --- & --- & ---- & --- & --- & \\
\hline \multirow{3}{*}{ Temporal } & Género & \multirow{3}{*}{$\begin{array}{c}4,188 \\
p=0,007\end{array}$} & $-7,571$ & $-0,268$ & $-3,129$ & $-12,012$ & 0,001 & \multirow{3}{*}{0,060} \\
\hline & Idade & & 0,354 & 0,084 & 1,031 & $-0,322$ & 0,302 & \\
\hline & ES & & $-0,678$ & $-0,064$ & 1,031 & $-2,387$ & 0,434 & \\
\hline \multirow{3}{*}{ Nasal } & Género & \multirow{3}{*}{$\begin{array}{c}3,704 \\
p=0,013\end{array}$} & $-6,105$ & $-0,204$ & $-1,378$ & $-10,832$ & 0,012 & \multirow{3}{*}{0,051} \\
\hline & Idade & & 0,629 & 0,141 & 1,350 & $-0,091$ & 0,086 & \\
\hline & ES & & $-1,156$ & $-0,103$ & 0,663 & $-2,975$ & 0,211 & \\
\hline \multicolumn{9}{|l|}{ Anel Externo } \\
\hline & Género & & ---- & --- & --- & --- & --- & \\
\hline Média global & Idade & $\begin{array}{c}1,377 \\
p=0,252\end{array}$ & --- & --- & --- & --- & --- & ---- \\
\hline & ES & & ---- & ---- & ---- & ---- & ---- & \\
\hline & Género & & 4,179 & 0,159 & 8,368 & $-0,010$ & 0,051 & \\
\hline Superior & Idade & $\begin{array}{c}2,874 \\
p=0,038\end{array}$ & $-0,693$ & $-0,177$ & $-0,055$ & $-1,331$ & 0,033 & 0,036 \\
\hline & ES & & 0,376 & 0,038 & 1,988 & $-1,236$ & 0,645 & \\
\hline & Género & & ---- & ---- & ---- & ---- & ---- & \\
\hline Inferior & Idade & $\begin{array}{c}1,653 ; \\
p=0,180\end{array}$ & ---- & --- & --- & --- & --- & --- \\
\hline & ES & & --- & ---- & --- & ---- & ---- & \\
\hline & Género & & ---- & ---- & --- & ---- & ---- & \\
\hline Temporal & Idade & $\begin{array}{c}2,211 ; \\
p=0,089\end{array}$ & ---- & --- & --- & --- & --- & ---- \\
\hline & ES & & ---- & ---- & ---- & ---- & ---- & \\
\hline & Género & & ---- & ---- & --- & ---- & ---- & \\
\hline Nasal & Idade & $\begin{array}{c}0,881 \\
p=0,453\end{array}$ & --- & --- & --- & --- & --- & ---- \\
\hline & ES & & ---- & ---- & ---- & ---- & --- & \\
\hline Volume $\left(\mathrm{mm}^{3}\right)$ & & & & & & & & \\
\hline & Género & & ---- & ---- & ---- & ---- & ---- & \\
\hline Volume total & Idade & $\begin{array}{c}0,473 \\
p=0,702\end{array}$ & --- & --- & ---- & --- & --- & ---- \\
\hline & ES & & ---- & ---- & ---- & ---- & ---- & \\
\hline
\end{tabular}

CC, campo central; EM, espessura macular; ES, equivalente esférico; IC, intervalo de confiança; LI, limite inferior; LS, limite superior; VI, variáveis independentes. 
ser interpretados com cuidado, pois o estudo de Wu et al só envolveu sujeitos com ES > -6 dioptrias, os diferentes estudos não são consensuais ${ }^{4,11,16,17}$ e o exacto mecanismo por detrás desta relação permanece por esclarecer. A idade apresentou também influência significativa, verificando-se, à semelhança com estudos prévios, ${ }^{11,14,16,17}$ um aumento da espessura macular do campo central com a idade, o que é consistente com estudos anatómicos que sugerem um desenvolvimento contínuo da fóvea para além dos 5 anos de idade. ${ }^{14}$

É de realçar que outras variáveis apresentam influência nos parâmetros obtidos pela OCT. A raça é um dos fatores largamente estudado e cujo impacto é reconhecido ao nível da espessura da CFNR ${ }^{15,36}$ e da espessura macular. ${ }^{11,36}$ Como neste estudo não foram incluídas crianças de outras raças é necessário cuidado na extrapolação dos resultados. Por outro lado, as normativas em adultos geralmente incluem a avaliação por perimetria, gonioscopia e determinação do comprimento axial. Além de ser reconhecido que a influência do comprimento axial em crianças caucasianas é reduzida, ${ }^{15}$ optou-se por não incluir avaliações de difícil execução nesta faixa etária para não limitar a participação no estudo. Outras limitações possíveis são a dimensão da amostra e o facto de todas as crianças terem sido recrutadas em consulta hospitalar, pelo qual a nossa população em estudo pode não refletir de modo preciso a população

\section{REFERÊNCIAS}

1. Budenz DL. Symmetry between the right and left eyes of the normal retinal nerve fiber layer measured with optical coherence tomography (an AOS thesis). Trans Am Ophthalmol Soc. 2008;106:252-75.

2. Schuman JS. Spectral domain optical coherence tomography for glaucoma (an AOS thesis). Trans Am Ophthalmol Soc. 2008;106:42658

3. Huynh SC, Wang XY, Rochtchina E, Mitchell P. Peripapillary retinal nerve fiber layer thickness in a population of 6-year-old children: findings by optical coherence tomography. Ophthalmology. 2006;113:1583-92.

4. Turk A, Ceylan OM, Arici C, Keskin S, Erdurman C, Durukan AH, et al. Evaluation of the nerve fiber layer and macula in the eyes of healthy children using spectral-domain optical coherence tomography. Am J Ophthalmol. 2012;153:552-9.

5. Zhang Z, He X, Zhu J, Jiang K, Zheng W, Ke B. Macular measurements using optical coherence tomography in healthy Chinese school age children. Invest Ophthalmol Vis Sci. 2011;52:6377-83.

6. Moreno-Montañés J, Olmo N, Alvarez A, García N, Zarranz-Ventura J. Cirrus high-definition optical coherence tomography compared with Stratus optical coherence tomography in glaucoma diagnosis. Invest Ophthalmol Vis Sci. 2010;51:335-43.

7. Leung MM, Huang RY, Lam AK. Retinal nerve fiber layer thickness in normal Hong Kong Chinese children measured with optical coherence tomography. J Glaucoma. 2010;19:95-9.

8. Qian J, Wang W, Zhang X, Wang F, Jiang Y, Wang W, et al. Optical coherence tomography measurements of retinal nerve fiber layer thickness in chinese children and teenagers. J Glaucoma. 2011;20:50913.

9. Altemir I, Pueyo V, Elía N, Polo V, Larrosa JM, Oros D. Reproducibility of optical coherence tomography measurements in children. Am J Ophthalmol. 2013;155:171-6.

10. Salchow DJ, Oleynikov YS, Chiang MF, Kennedy-Salchow SE, Langton K, Tsai JC, et al. Retinal nerve fiber layer thickness in normal children measured with optical coherence tomography. Ophthalmology. 2006;113:786-91.

11. Huynh SC, Wang XY, Rochtchina E, Mitchell P. Distribution of macular thickness by optical coherence tomography: findings from a populationbased study of 6-year-old children. Invest Ophthalmol Vis Sci. 2006;47:2351-7. pediátrica em geral. Para minimizar este viés de seleção foram estabelecidos rigorosos critérios de inclusão e exclusão. No futuro seria essencial estudos com maior número de crianças, estratificadas por grupo etário, género e raça e averiguação da influência de outras variáveis na espessura da CFNR e espessura macular.

\section{CONCLUSÃO}

Tanto quanto foi possível apurar, este é o primeiro estudo a estabelecer as normativas da espessura da CFNR e espessura macular em crianças portuguesas saudáveis dos 4 aos 17 anos de idade por recurso ao Cirrus SD-OCT. Esta informação tem potencial para reestruturar a avaliação e interpretação dos parâmetros obtidos pela OCT no diagnóstico de patologias pediátricas na prática clínica, na qual será imprescindível ter particular consideração com o género, raça e erro refrativo da criança.

\section{CONFLITO DE INTERESSES}

Os autores declaram não ter qualquer conflito de interesse relacionado com a realização deste trabalho.

\section{FONTES DE FINANCIAMENTO}

Os autores declaram não ter recebido qualquer subsídio relativo ao presente artigo.

12. El-Dairi MA, Asrani SG, Enyedi LB, Freedman SF. Optical coherence tomography in the eyes of normal children. Arch Ophthalmol. 2009;127:50-8

13. Eriksson U, Holmström G, Alm A, Larsson E. A population-based study of macular thickness in full-term children assessed with Stratus OCT: normative data and repeatability. Acta Ophthalmol. 2009;87:741-5.

14. Yanni SE, Wang J, Cheng CS, Locke KI, Wen Y, Birch DG, et al Normative reference ranges for the retinal nerve fiber layer, macula, and retinal layer thicknesses in children. Am J Ophthalmol. 2013;155:35460.

15. Elía N, Pueyo V, Altemir I, Oros D, Pablo LE. Normal reference ranges of optical coherence tomography parameters in childhood. $\mathrm{Br} J$ Ophthalmol. 2012;96:665-70.

16. Al-Haddad C, Barikian A, Jaroudi M, Massoud V, Tamim H. Spectral domain optical coherence tomography in children: normative data and biometric correlations. BMC Ophthalmology. 2014;14:53.

17. Barrio-Barrio J, Noval S, Galdos M, Ruiz-Canela M, Bonet E, Capote M, et al. Multicenter Spanish study of spectral-domain optical coherence tomography in normal children. Acta Ophthalmol. 2013;91:e56-63.

18. Chia A, Jaurigue A, Gazzard G, Wang Y, Tan D, Stone RA, et al. Ocular dominance, laterality, and refraction in Singaporean children. Invest Ophthalmol Vis Sci. 2007;48:3533-6.

19. Carl Zeiss Meditec. Cirrus HD-OCT Model 4000 Documentation Set. 2009.

20. El-Dairi MA, Holgado S, O'Donnell T, Buckley EG, Asrani S, Freedman SF. Optical coherence tomography as a tool for monitoring pediatric pseudotumor cerebri. J AAPOS. 2007;11:564-70.

21. Medeiros FA, Moura FC, Vessani RM, Susanna Jr R. Axonal loss after traumatic optic neuropathy documented by optical coherence tomography. Am J Ophthalmol. 2003;135:406-8.

22. Seibold LK, Mandava N, Kahook MY. Comparison of retinal nerve fiber layer thickness in normal eyes using time-domain and spectral-domain optical coherence tomography. Am J Ophthalmol. 2010;150:807-14

23. Huang J, Liu X, Wu Z, Guo X, Xu H, Dustin L, et al. Macular and retinal nerve fiber layer thickness measurements in normal eyes with the Stratus OCT, the Cirrus HD-OCT, and the Topcon 3D OCT-1000. J Glaucoma. 2011:20:118-25

24. Kakinoki M, Sawada O, Sawada T, Kawamura H, Ohji M. Comparison 
of macular thickness between Cirrus HD-OCT and Stratus OCT. Ophthalmic Surg Lasers Imaging. 2009;40:135-40.

25. Huynh SC, Wang XY, Burlutsky G, Mitchell P. Symmetry of optical coherence tomography retinal measurements in young children. Am J Ophthalmol. 2007;143:518-20.

26. Mwanza JC, Durbin MK, Budenz DL. Interocular symmetry in peripapillary retinal nerve fiber layer thickness measured with the Cirrus HD-OCT in healthy eyes. Am J Ophthalmol. 2011;151:514-21.

27. Hoh ST, Lim MC, Seah SK, Lim AT, Chew SJ, Foster PJ, et al. Peripapillary retinal nerve fiber layer thickness variations with myopia. Ophthalmology. 2006;113:773-7.

28. Wang XY, Huynh SC, Burlutsky G, Ip J, Stapleton F, Mitchell P. Reproducibility of and effect of magnification on optical coherence tomography measurements in children. Am J Ophthalmol. 2007;143:4848.

29. Huang D, Chopra V, Lu AT, Tan O, Francis B, Varma R. Does optic nerve head size variation affect circumpapillary retinal nerve fiber layer thickness measurement by optical coherence tomography? Invest Ophthalmol Vis Sci. 2012;53:4990-7.

30. Tariq YM, Burlutsky G, Mitchell P. Macular parameters and prematurity: A spectral domain coherence tomography study. J AAPOS. 2012;16:3825 .
31. Ooto S, Hangai M, Sakamoto A, Tomidokoro A, Araie M, Otani T, et al. Three-dimensional profile of macular retinal thickness in normal Japanese eyes. Invest Ophthalmol Vis Sci. 2010;51:465-73.

32. Song WK, Lee SC, Lee ES, Kim CY, Kim SS. Macular thickness variations with gender, age, and axial length in healthy subjects, a spectral domain optical coherence tomography study. Invest Ophthalmol Vis Sci. 2010;51:3913-8.

33. Samarawickrama C, Wang JJ, Huynh SC, Wang XY, Burlutsky G, Stapleton F, et al. Macular thickness, retinal thickness, and optic disk parameters in dominant compared with nondominant eyes. J AAPOS. 2009;13:142-7.

34. Azzolini C, Patelli F, Brancato R. Correlation between optical coherence tomography data and biomicroscopic interpretation of idiopathic macular hole. Am J Ophthalmol. 2001;132:348-55.

35. Wu P, Chen Y, Chen C, Chen Y, Shin S, Yang H, et al. Assessment of macular retinal thickness and volume in normal eyes and highly myopic eyes with third-generation optical coherence tomography. Eye. 2007;22:551-5.

36. Girkin CA, McGwin Jr G, Sinai MJ, Sekhar GC, Fingeret M, Wollstein G, et al. Variation in optic nerve and macular structure with age and race with spectral-domain optical coherence tomography. Ophthalmology. 2011;118:2403-8 
Tatiana QUEIRÓS, Cristina FREITAS, Sandra GUIMARÃES

\section{Valores de Referência da Tomografia de Coerência Óptica na Idade Pediátrica}

Acta Med Port 2015:28:148-157

Publicado pela Acta Médica Portuguesa, a Revista Científica da Ordem dos Médicos

Av. Almirante Gago Coutinho, 151

1749-084 Lisboa, Portugal.

Tel: +351218428215

E-mail: submissao@actamedicaportuguesa.com

www.actamedicaportuguesa.com

ISSN:0870-399X | e-ISSN: 1646-0758

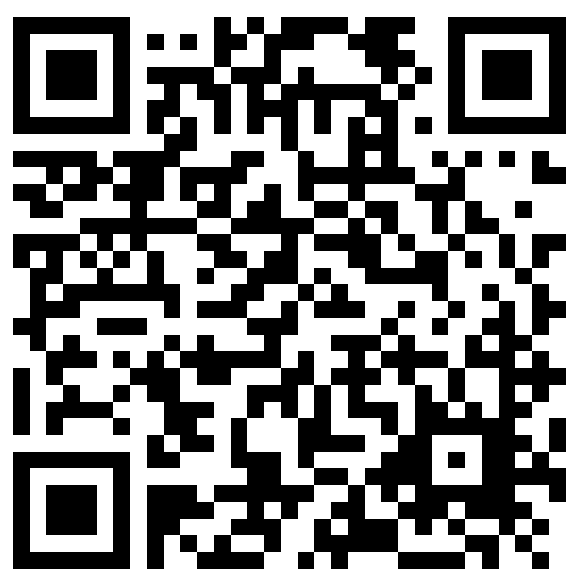

